\title{
Spin Probing and Labeling in Physical Chemistry
}

\author{
Alexander I. Kokorin ${ }^{1}$ - Wolfgang E. Trommer ${ }^{2}$. \\ Howard J. Halpern ${ }^{3}$
}

A special class of chemical reactions, in which the unpaired electrons of stable radicals did not react, was opened 50 years ago (M. B. Neiman and E. G. Rozantzev 1964). These nitroxide (aminoxyl) radicals, after half a century, have become among the most applicable and highly investigated objects of modern organic and physical chemistry, in molecular biology, biophysics, polymer sciences and medicine. Nitroxide radicals of different types are widely used in EPR spectroscopy particularly for measuring large molecule intramolecular distances, as shifting reagents in NMR, as $\mathrm{pH}$-sensitive and target spin labels, probes and spin traps (and their precursors), for in vivo oximetry, for constructing supramolecular systems and molecular magnets, supermagnetics, etc.

Further development of this field depends on the development of new pathways in nitroxide synthesis and modern methods in EPR spectroscopy revealing new perspectives and practical approaches. Articles of this issue illustrate new results and perspectives using nitroxide radicals in the areas of chemistry of $\beta$ phosphorylated nitroxides and selectively deuterated nitronyl nitroxides, in situ EPR detection of ROS in adherent cells, spin probe investigation of smectic liquid

$\triangle$ Alexander I. Kokorin

alex-kokorin@yandex.ru

$\bowtie$ Wolfgang E. Trommer trommer@chemie.uni-kl.de

$\bowtie$ Howard J. Halpern

h-halpern@uchicago.edu

1 Department of Kinetics and Catalysis, N.N. Semenov Institute of Chemical Physics, Russian Academy of Sciences, Kosygin st. 4, 119991 Moscow, Russian Federation

2 Department of Chemistry, Technical University of Kaiserslautern, P. O. Box 3049, 67653 Kaiserslautern, Germany

3 Department of Radiation and Cellular Oncology, University of Chicago, Medical Center, 5841 S. Maryland Ave., Chicago, IL 60637, USA 
crystals, electrosurface properties of nanostructured silica, sorbitol and poly(diphenilenesulfophtalide) hydrogels, the shungite/elastomer interfaces, for studying of cationic dinitrosyl iron salts and complexes. Nitroxide biradicals were used for investigation of the structure and properties of molecules containing a phosphorus atom and the influence of pressure on the intramolecular dynamics.

We hope that this issue will be of help to chemists, physicists, biologists and other experts who use spin labels, probes and traps in their studies, as well as to graduate and/or $\mathrm{PhD}$ students. 